\title{
Crack propagation analysis and rehabilitation by HFMI of pre-fatigued welded structures
}

\author{
M. Leitner ${ }^{1} \cdot$ Z. Barsoum ${ }^{2} \cdot$ F. Schäfers ${ }^{3}$
}

Received: 5 October 2015 / Accepted: 16 February 2016 / Published online: 8 March 2016

(C) The Author(s) 2016. This article is published with open access at Springerlink.com

\begin{abstract}
This paper deals with a crack propagation analysis of welded structures and rehabilitation after pre-fatigue loading by the high-frequency mechanical impact (HFMI) posttreatment technique. The investigated specimen type is a thinwalled longitudinal stiffener made of mild steel $S 355$. Fracture mechanical calculations are primarily performed on the basis of the weight-function approach. Thereby, the local residual stress condition at the weld toe is considered by the aid of a structural weld simulation, whereas the numerically evaluated residual stress distribution in depth agrees well to X-ray measurement results. The fracture mechanical analysis illustrates that by incorporating the residual stress state, the calculated lifetime is in good accordance to the conducted fatigue test results. By application of the HFMI-treatment as rehabilitation method, it is found that the beneficial post-treatment effect increases especially by a reduction of the applied load-level. Hence, particularly for minor nominal stress ranges near the high-cycle fatigue region, the mechanical post-treatment as repair method is utmost effective leading to almost equal fatigue strength as for the HFMI-treated specimens without pre-cycling. Finally, proposals for the crack growth
\end{abstract}

Recommended for publication by Commission XIII - Fatigue of Welded Components and Structures

M. Leitner

martin.leitner@unileoben.ac.at

1 Department Product Engineering, Chair of Mechanical Engineering, Montanuniversität Leoben, Leoben, Austria

2 KTH-Royal Institute of Technology, Department of Aeronautical and Vehicle Engineering, Division of Lightweight Structures, Stockholm, Sweden

3 Pitec GmbH, Heudorf, Germany assessment of welded structures and a conservative application of HFMI as rehabilitation method for mild steel joints are provided.

Keywords (IIW Thesaurus) Crack propagation . Fatigue loading · Fatigue improvement · Weld toes . Residual stresses $\cdot$ Mechanical stress relief · Stress distribution $\cdot$ Post weld operations $\cdot$ Computation . Structural members $\cdot$ Fracture mechanics

\section{Introduction}

To assess the fatigue strength of welded joints, recommendations [1] are available, which involve different stress-based approaches, such as the nominal, the structural and the effective notch stress method. However, it is well known that the fatigue strength of welded parts is significantly affected by local properties, such as the local geometry at the weld toe or root and the residual stress condition. In addition, discontinuities like flaws, undercuts or pores cannot be avoided during gas-metal arc welding and need to be statistically considered in the fatigue assessment, whereas fracture mechanical procedures based on an evaluation of the linear-elastic crack propagation are well suited for this kind of task [2,3]. An important application area of this concept occurs in case of large structures, where often additional service life is granted by maintaining recurring service intervals. If a crack is then non-destructively detected [4], different kinds of posttreatment techniques are feasible, at which one efficient method is offered by the high-frequency mechanical impact (HFMI) treatment due to a feasible and expeditious application on site. Recently, numerous investigations $[5,6]$ showed the beneficial effect of this method for an application without 


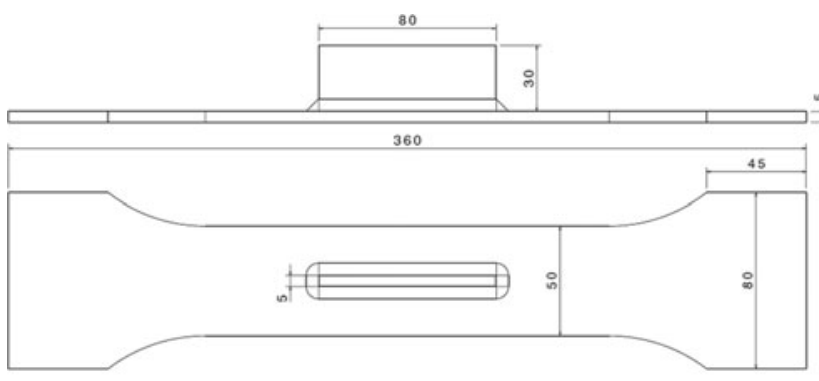

Fig. 1 Geometry of investigated longitudinal stiffener specimen

any cyclic pre-loading, but however, the application as rehabilitation purpose is not sufficiently investigated yet.

As a proper assessment of the crack propagation during operation and the specific conditions to extend the lifetime is of great importance, this paper contributes to the crack propagation analysis of welded joints and the subsequent rehabilitation of pre-fatigued structures by the HFMI post-treatment, see chapter 6.2. As the fatigue crack growth regime is predominant in case of notched joints, a longitudinal stiffener specimen made of mild steel $S 355$ is investigated (Fig. 1).

\section{Fatigue tests}

At first, fatigue tests in the as-welded and HFMI-treated conditions without any pre-fatigue of the specimens are performed. The stress ratio is set to $R=0.1$, and the abort criterion is burst fracture. The evaluated nominal a/ $\mathrm{N}$ curves are presented in Fig. 2 exhibiting a survival probability of $P_{S}=97.7 \%$.

The results show that the HFMI-treatment increases the fatigue strength especially in the high-cycle fatigue region

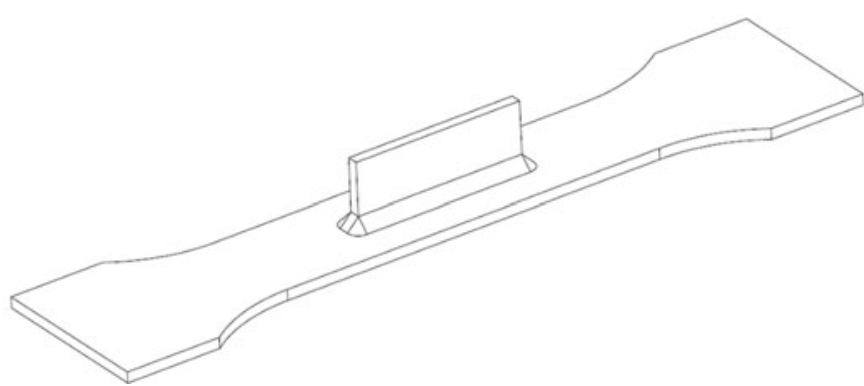

due to a shift of the transition knee point to a lower lifetime. The nominal stress values of the HFMI-treated specimens at the run-out level at fifty million load-cycles exceed with $\Delta \sigma_{n}=200 \mathrm{MPa}$ the as-welded condition by a factor of two. In the finite lifetime regime, an improvement is still observable, mainly due to an increase of the slope from $k=5.1$ for the as-welded to $k=9.2$ for the post-treated specimens. A comparison to the recommended [1] curve for the as-welded and the proposed [7] curve for the HFMI-treated condition indicates a high welding and post-treatment quality which fulfil the recommended demands [8].

For an analysis of the technical crack initiation and the subsequent macroscopic crack propagation, strain gauges at the weld toe region of the end-of-seam areas are applied and optical measurements by the aid of a zinc-oxide paste as contrast medium are performed, see Fig. 3. A sensitivity study involving numerous tests leads to the relation between the decrease of the maximum measured strain gauge values at the weld toe, the optically evaluated surface crack, and to the corresponding crack depth based on micrographs of the pre-fatigued specimens. In Fig. 3a, a drop of about $20 \%$ of the initial stress $\sigma_{\max , 1}$ down to $\sigma_{\max , 2}$ is observed. The attendant
Fig. 2 Nominal a/N curves for as-welded and HFMI-treated conditions

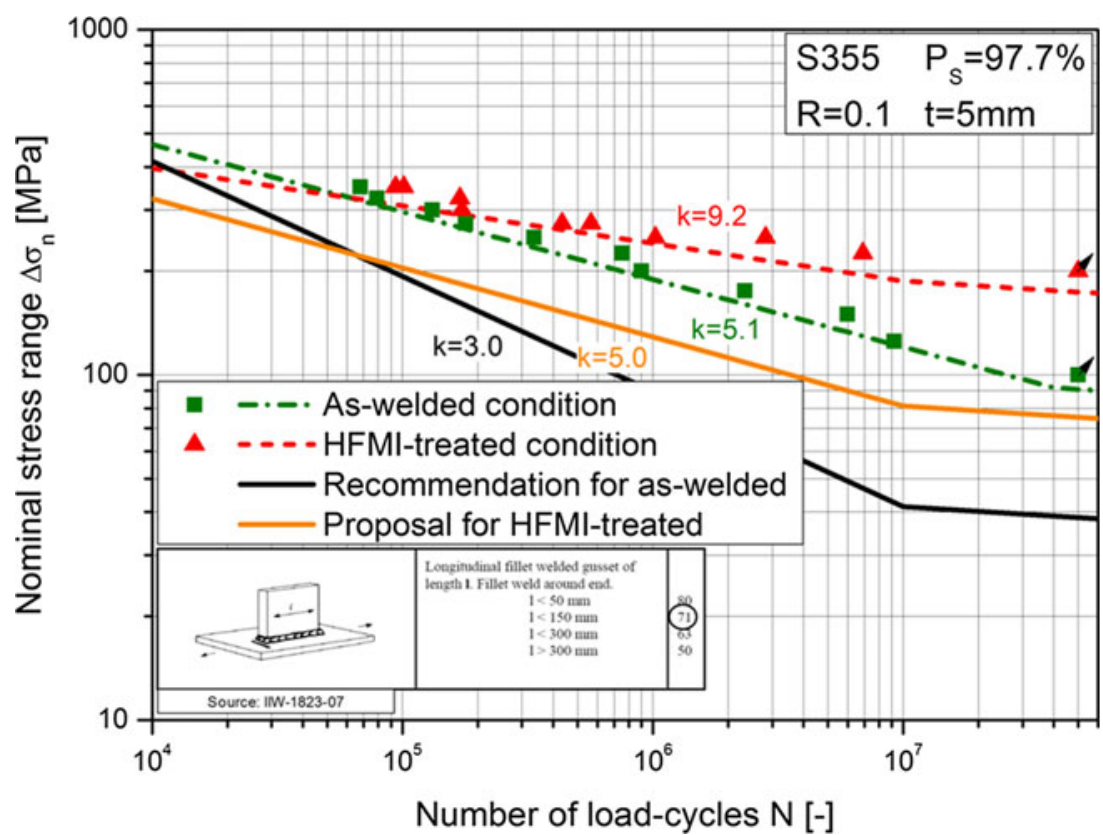



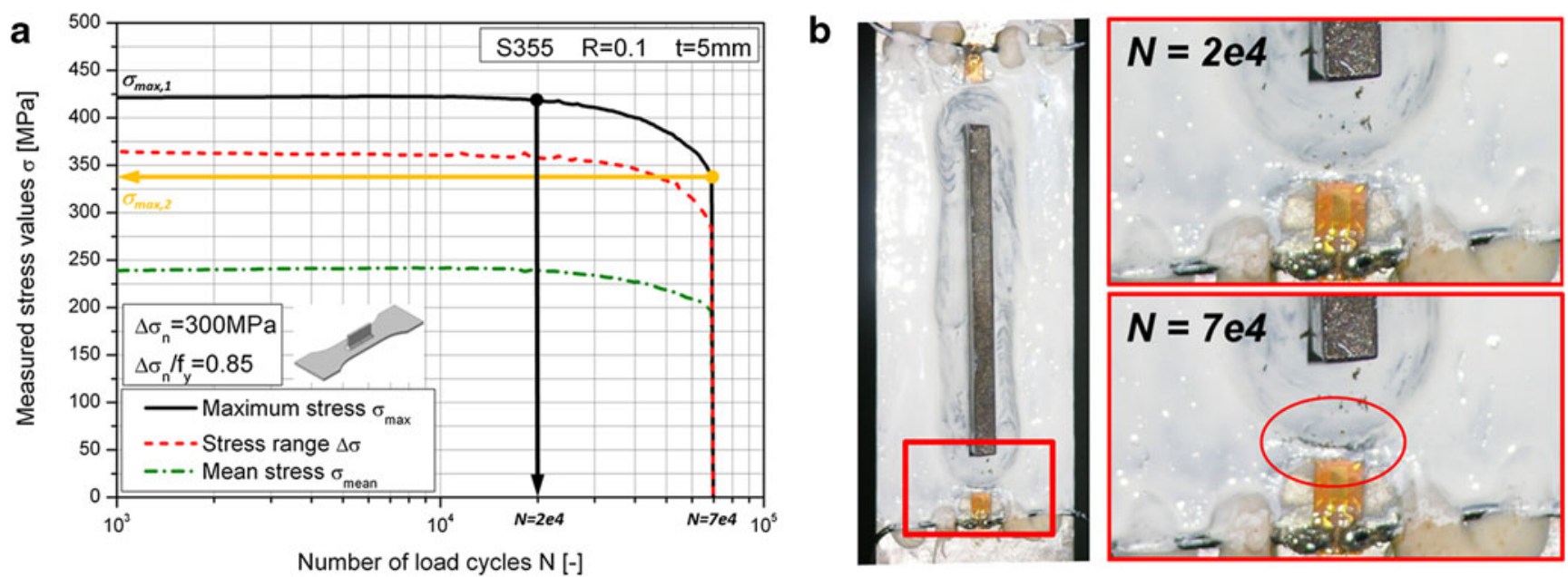

Fig. 3 a Strain gauge measurements and $\mathbf{b}$ optical surface crack detection [9]

optical evaluation of the surface crack behaviour for the two corresponding fatigue-lives in Fig. 3b shows a crack initiation at $N=7 \mathrm{e} 4$ and $\sigma_{\max , 2}$ at which the fatigue testing is afterwards stopped. Detailed information regarding the fatigue tests and strain gauge measurements is provided in [9].

Based on the presented experimental procedure, a precycling of the specimens in the as-welded condition up to a defined crack depth is enabled, see also [10]. In Fig. 4, the resulting pre-fatigued $\mathrm{a} / \mathrm{N}$ curve implying a crack depth of about $a=1 \mathrm{~mm}$ in comparison to the previously depicted tests up to burst fracture is evaluated by a survival probability of $P_{S}=50 \%$. Thereby, only a minor difference is noticeable, which is caused by the fact that the bulk of the total lifetime is spent in the small crack size region. Experimental and numerical investigations in [11] show that about $80 \%$ of the total fatigue-life is spent to grow the crack up to $20 \%$ of the related wall thickness, which basically proves the results in this paper. The pre-fatigued specimens are further on used to assess the potential of the HFMI treatment as rehabilitation method.

\section{Local stress condition}

For the evaluation of the local notch stress distribution in depth at the weld toe, a quarter-symmetric model with a hexahedral element mesh and a unified tension load is setup. The modelled geometry depicts the nominal cross section
Fig. 4 Comparison of test results with specimens up to burst fracture and crack size $a=1 \mathrm{~mm}$

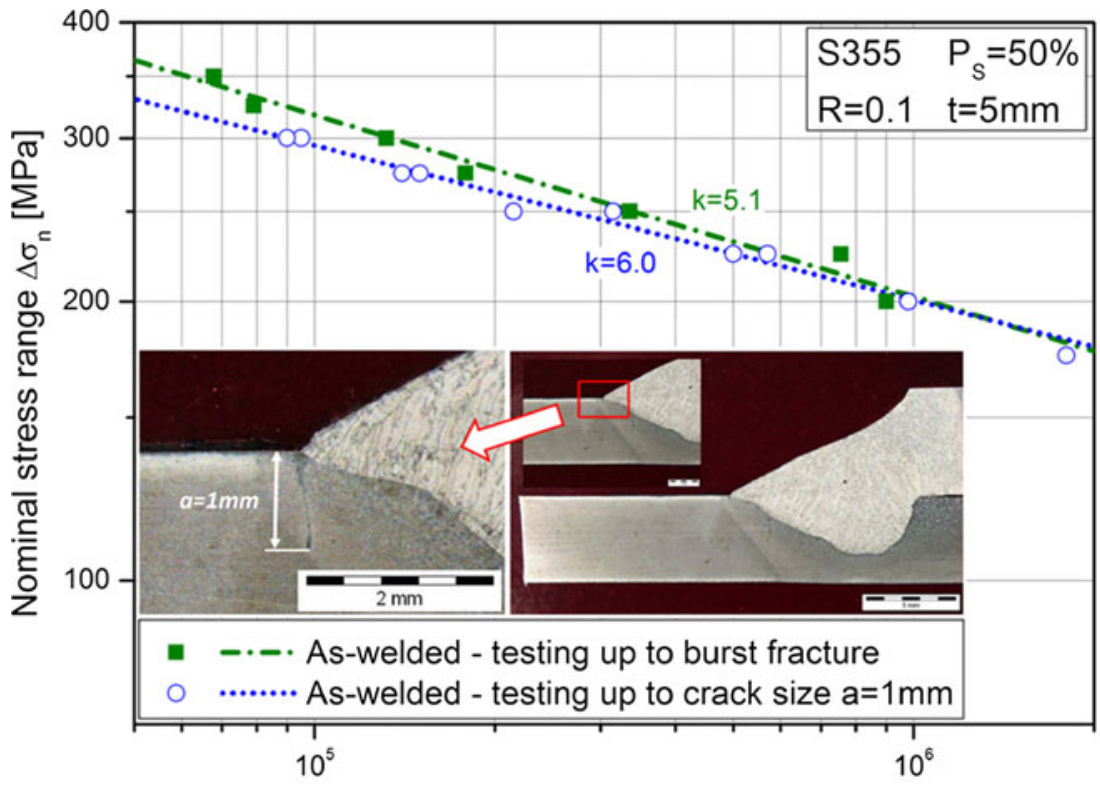

Number of load-cycles N [-] 
of the specimens, and local deviations from the design geometry are not incorporated in detail. Distortion measurements of the specimens before fatigue testing exhibit only minor values up to one-tenth-degree angular deformation. A further evaluation of the influence factor $k_{m}$ given in [1] shows almost no impact, and therefore, an explicit consideration in the numerical analysis is not necessary. The mesh is built-up in accordance to the recommended values for the effective notch stress assessment in [12] and is adapted to study the effect of varying weld toe radii. The stress evaluation is performed for the component $S 11$ appearing in loading direction of the specimen and therefore most significant for the subsequent crack propagation analysis, see Fig. 5a.

In Fig. 5b, the results of the numerical stress analysis are depicted. Thereby, the local stress distribution in depth in dependency of the weld toe radius, ranging from $\rho=0.05$ to $2 \mathrm{~mm}$, is presented. It is shown that at the surface region up to a depth of about $0.1 \mathrm{~mm}$, the weld toe radius has a major influence on the local stress condition. Especially for small radii of about $\rho=0.05$ up to $0.2 \mathrm{~mm}$, a high stress concentration is evaluated.

\section{Structural weld simulation}

In order to improve the accuracy of the crack propagation results, also the residual stress condition needs to be incorporated. In general, residual stresses can be determined by means of measurements or numerical analysis. Basic investigations involving a structural weld simulation of the investigated joint type and material are shown in [13]. Thereby, an enhanced method to incorporate the transient work-hardening effect of different material phases into a thermo-mechanically coupled structural weld process simulation is presented, whereas the work-hardening behaviour of metals principally differs for soft ferritic-perlitic and hard bainitic, or even martensitic, phases. For the conducted thermo-mechanical coupled weld process simulation, the software package Sysweld [14] is utilized, which supports the implementation of different workhardening models, such as the basic isotropic behaviour and the kinematic hardening rule according to [15]. Moreover, it is possible to apply the kinematic portion [16], which takes, besides the effect of backstress, the influence of dynamic recovery during cyclic loading into account [17].

One of the main drawbacks of the described material models is that they are basically not able to consider the effect of phasechange during the transient weld simulation. This means, that the unique material property database is defined at the stage of input card definition and cannot be easily modified for each element possessing process time- and temperature-dependent different hardening parameters, see [13].

To solve the task of transient change in work-hardening per element during the numerical simulation process, a userdefined SIL routine was previously developed [18]. It enables the implementation of a temperature- and phase-dependent work-hardening behaviour during the transient thermomechanical weld simulation. Based on low-cycle-fatigue test results, three different work-hardening behaviours are classified, which depend on the temperature and the major phase content, separated in mainly ferritic-perlitic as soft microstructure; predominantly bainitic-martensitic as hard phase or mixed microstructure. At ambient temperature, the kinematic behaviour is more appropriate, whereas at higher temperatures, close to the austenitic region, the isotropic workhardening rule is more applicable. The results of the mixed material model calculate the residual stress condition quite well [13]. These results are applied for the investigations in this paper. In Fig. 6a, the conducted structural weld simulation model and the temperature distribution during welding is displayed. a

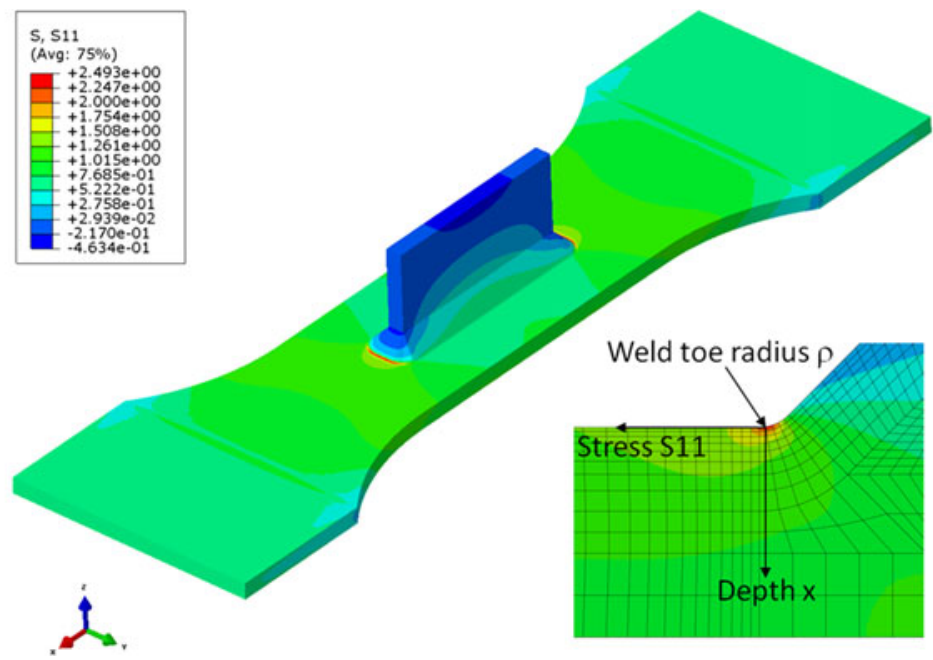

b

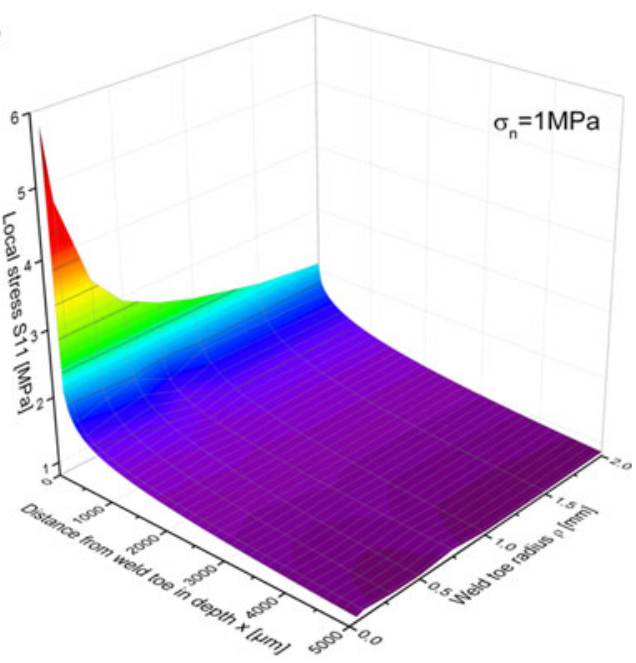

Fig. 5 a Numerical model for analysis of local stress condition and $\mathbf{b}$ in-depth notch stress distribution in dependency of weld toe radius 


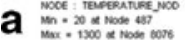

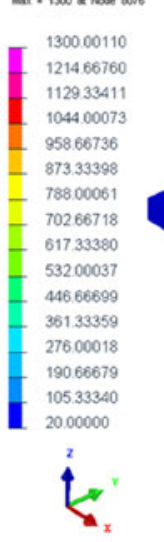

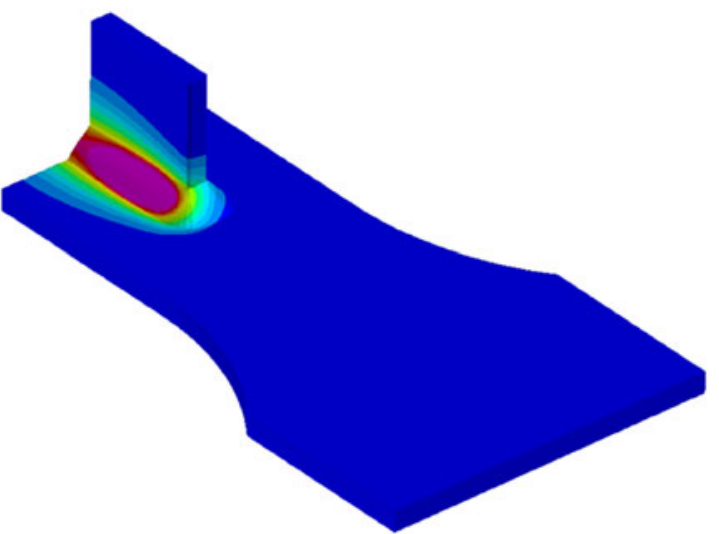

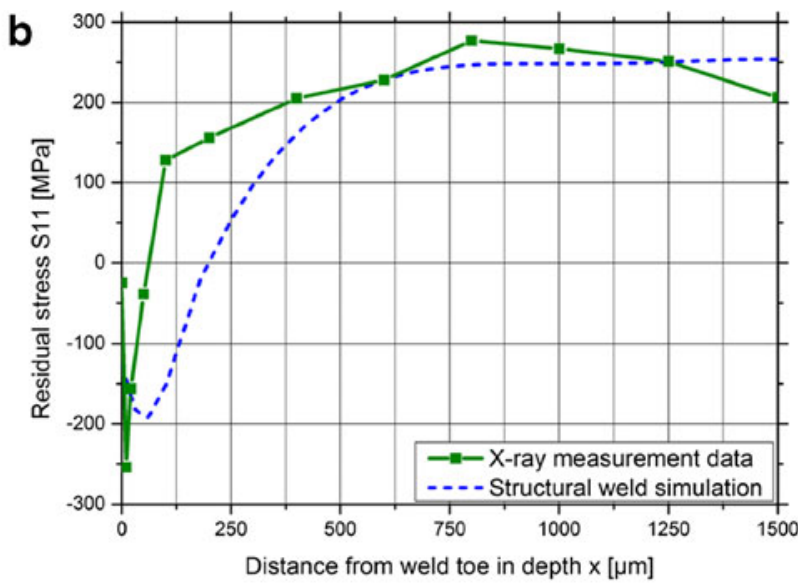

Fig. 6 a Temperature distribution during welding and $\mathbf{b}$ comparison of measured and simulated residual stress values

In Fig. 6b, the numerically evaluated residual stress values $S 11$ in depth are compared to experimental data by X-ray measurements for the as-welded condition. Additional residual stress measurements in [9] focusing on the base material exhibit a major compressive residual stress state in the surface layer of the metal sheet, which primarily emerge due to the base-material rolling process. By a superposition of these compressive stresses in the numerical weld simulation, the results in the as-welded condition show a good compliance to the measured values and are therefore used for the subsequent crack propagation analysis.

\section{Crack propagation analysis}

Fracture mechanical approaches provide the basis for the assessment of cracks or crack-like imperfections, where the crack propagation significantly exceeds the crack initiation phase, see [19]. For welded joints, the basic elements of the linear-elastic crack growth calculation are the stress intensity factor $K$, the crack shape and path and the chosen material parameters, see Table 1 . The crack propagation is mostly calculated based on the simple power law according to Paris and Erdogan [21]:

$\frac{d a}{d N}=C \cdot \Delta K^{m}$ for $\Delta K>\Delta K_{t h}$

Table 1 Recommended crack propagation data for steel [20]

Paris power law parameters Threshold values $\Delta K_{\text {th }}[\mathrm{MPa} \sqrt{\mathrm{m}}]$ (charact. values)

$R>0.5 \quad 0 \leq R \leq 0.5 \quad R<0 \quad$ Surface crack depth $<1 \mathrm{~mm}$

$C=9.5 \cdot 10^{-12}$
$m=3.0$
2.0

For standardized crack geometries and load configurations, numerous stress intensity factor formulae are available [22]. However, there are basically no ready-made solutions for welded structures except for a few crack configurations. Therefore, the weight function technique [23, 24] was employed in order to determine stress intensity factors for cracks in real welded structures, see [25].

\subsection{Weight-function approach}

The weight function approach is basically defined as the stress intensity factor $K$ induced by the simplest load configuration, such as a pair of unit forces $F$ attached to the crack surface, see Fig. 7. The operation of each differential force on a crack is specified by the weight-function $[20,25]$.

A general form to describe the weight function is provided by [24] according to Eq. 2 . The containing parameters $\mathrm{M}_{1}$, $\mathrm{M}_{2}$, and $\mathrm{M}_{3}$ depend on the actual crack geometry and are listed in $[24,25]$ for numerous crack configurations. For the crack propagation analysis of the investigated longitudinal

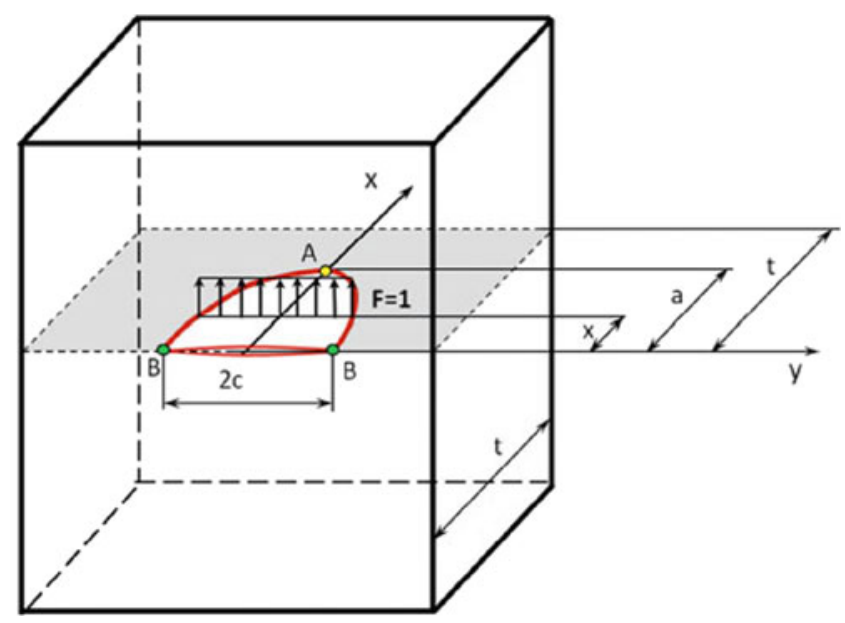

Fig. 7 Semi-elliptical surface crack in plate [26] 
stiffener in this paper, the accordant expressions for a semielliptical crack at the deepest point A are applied.

$m(x, a)=\frac{2 \cdot F}{\sqrt{2 \cdot \pi \cdot(a-x)}} \cdot\left[1+M_{1} \cdot\left(1-\frac{x}{a}\right)^{\frac{1}{2}}+M_{2} \cdot\left(1-\frac{x}{a}\right)+M_{3} \cdot\left(1-\frac{x}{a}\right)^{\frac{3}{2}}\right]$

To calculate the stress intensity factor $K$, the knowledge of the stress distribution $\sigma(x)$ in the prospective crack plane of the un-cracked plate is required. In [20], it is recommended to involve the stress distribution assuming a weld toe radius of $\rho=0$ to $0.2 \mathrm{~mm}$, which is already computed within the local stress analysis depicted in "Local stress condition". Further on, the stress intensity factor $K$ is calculated by integrating the product of the stress distribution $\sigma(x)$ and the weight function $m(x, a)$ for each crack increment during cyclic loading, see Eq. 3.

$K=\int_{0}^{a} \sigma(x) \cdot m(x, a) \cdot d x$

To calculate the fatigue-life more precisely, the influence of the residual stress condition at the vicinity of the crack at the weld toe has to be considered. Based on the numerically evaluated residual stress condition, a procedure based on investigations in [27] involving an effective stress ratio $R_{\text {eff }}$ [28] at the crack tip, is applied, see Eq. 4.

$\frac{d a}{d N}=C \cdot\left(\frac{\Delta K}{1.5-R_{\mathrm{eff}}}\right)^{m}$

The stress intensity factor range $\Delta K$ is defined by the difference of the maximum and minimum stress intensity factors at the crack tip and is hereby not affected by the residual stress state, see Eq. 5.

$$
\begin{aligned}
\Delta K & =K_{\max }-K_{\min } \text { with } K_{\max / \min } \\
& =\int_{0}^{a} \sigma_{\max } / \min (x) \cdot m(x, a) \cdot d x
\end{aligned}
$$

In accordance to the loading-dependent stress intensity factors $K_{\max }$ and $K_{\min }$, the residual stress intensity factor $K_{\text {res }}$ is calculated in the same way, see Eq. 6. The residual stress distribution in depth $\sigma_{\text {res }}(x)$ is derived from the result of the structural weld simulation.

$K_{\mathrm{res}}=\int_{0}^{a} \sigma_{\mathrm{res}}(x) \cdot m(x, a) \cdot d x$

Finally, the effective stress ratio $R_{\text {eff }}$ is defined as shown in Eq. 7. Thereby, the local stress ratio at the crack tip is influenced by the local residual stress condition and in this way incorporated in the crack propagation analysis and fatigue-life assessment.

$R_{\text {eff }}=\frac{K_{\min }+K_{\text {res }}}{K_{\max }+K_{\text {res }}}$

Fracture mechanical calculations are performed in accordance to the presented weight-function procedure for the longitudinal stiffener specimen. For the crack shape, the aspect ratio $a / c$ is one important influence factor, whereas general applicable recommendations are provided in [20]. As a conservative approach, a ratio of $a / c=0.1$ may be defined, but the calculation results exhibited that the computed lifetime is significantly lower than the fatigue test results. Therefore, the proposed formula for ends of longitudinal stiffeners in [20] is applied (Eq. 8), leading to a/c values of 0.7 to 0.8 , which are also in good accordance to the test results of the fractured specimens.

$c=\frac{6.71+2.58 \cdot a}{2}$

A comparison of the calculated $N_{\text {calc }}$ and experimentally evaluated load-cycles $N_{\text {exp }}$ is presented in Fig. 8 as normalized ratio $N_{\text {calc }} / N_{\text {exp }}$ in dependency of the initial crack length $a_{i}$ and the weld toe radius $\rho$. Basically, it is shown that not only the initial crack length value of $a_{i}=0.1 \mathrm{~mm}$ is generally well applicable but also values of $a_{i}=0.05 \mathrm{~mm}$ which are suggested for mechanical engineering applications or $a_{i}=0.15$ $\mathrm{mm}$ as a conservative approach by fitness for purpose codes [20] are acceptable. However, to analyse the influence of the initial crack length in detail, a variation of $a_{i}=0.05$ up to $a$ ${ }_{i}=0.2 \mathrm{~mm}$ is included. In the finite lifetime regime at a nominal stress ratio of $\Delta \sigma_{n}=200 \mathrm{MPa}$, the calculated load-cycles are affected by the weld toe radius only in case of minor initial crack lengths of $a_{i}=0.05 \mathrm{~mm}$, leading to a significant overestimation of the lifetime, see Fig. 8a. For higher initial crack values, the influence of the weld toe radius is consequently decreasing.

On the contrary, in the high-cycle fatigue region at a nominal stress ratio of $\Delta \sigma_{n}=100 \mathrm{MPa}$, the minimum investigated initial crack length tends to result in good accordance to the fatigue tests incorporating weld toe radii smaller than $\rho=0.2 \mathrm{~mm}$, see Fig. 8b. Assuming a higher notch radius, the calculated lifetime is overestimated up to a factor of eight and is, therefore, not applicable. Summarized, the calculated lifetime $N_{\text {calc }}$ is strongly influenced by the weld toe radius, especially at a minor defined initial crack length of about $a_{i}=0.05 \mathrm{~mm}$.

As the calculated lifetime is significantly affected by the initial crack length and the weld toe radius at the high-cycle fatigue region, a detailed analysis of the crack tip loading involving a nominal stress of $\Delta \sigma_{n}=100 \mathrm{MPa}$ is performed, see Fig. 9. Thereby, the influence of the weld toe radius on the stress intensity factor range $\Delta K$ in dependency of the actual 

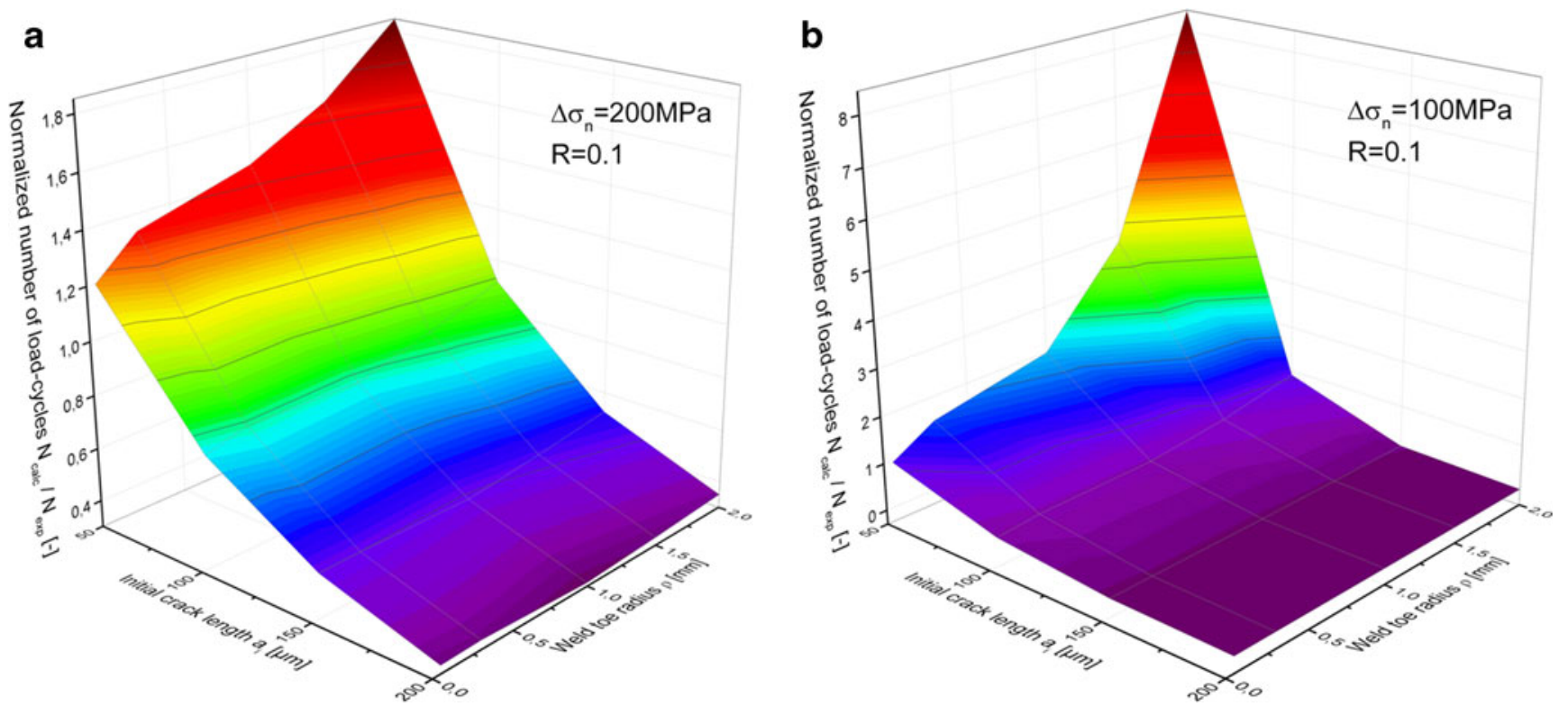

Fig. 8 Normalized number of load-cycles $N_{\text {calc }} / N_{\text {exp }}$ at a nominal stress range of $\mathbf{a} \Delta \sigma_{n}=200 \mathrm{MPa}$ and $\mathbf{b} \Delta \sigma_{n}=100 \mathrm{MPa}$

crack length $a$ is quite small, see Fig. 9a. Especially if the crack is technically propagated and not influenced by the stress concentration at the weld toe region anymore, the impact of the weld toe radius and the associated stress distribution on the stress intensity factor is negligible. Figure $9 \mathrm{~b}$ displays the stress intensity factor range $\Delta K$ related to the minimum recommended [20] threshold value of $\Delta K_{\mathrm{th}, \min }=2 \mathrm{MPa} \sqrt{\mathrm{m}}$ in dependence of the initial crack length $a_{i}$ and the weld toe radius $\rho$. One can see that especially for minor values of $a$ ${ }_{i}<0.1 \mathrm{~mm}$, the acting $\Delta K$ is below the threshold value and therefore, basically no long-crack-based crack propagation occurs.

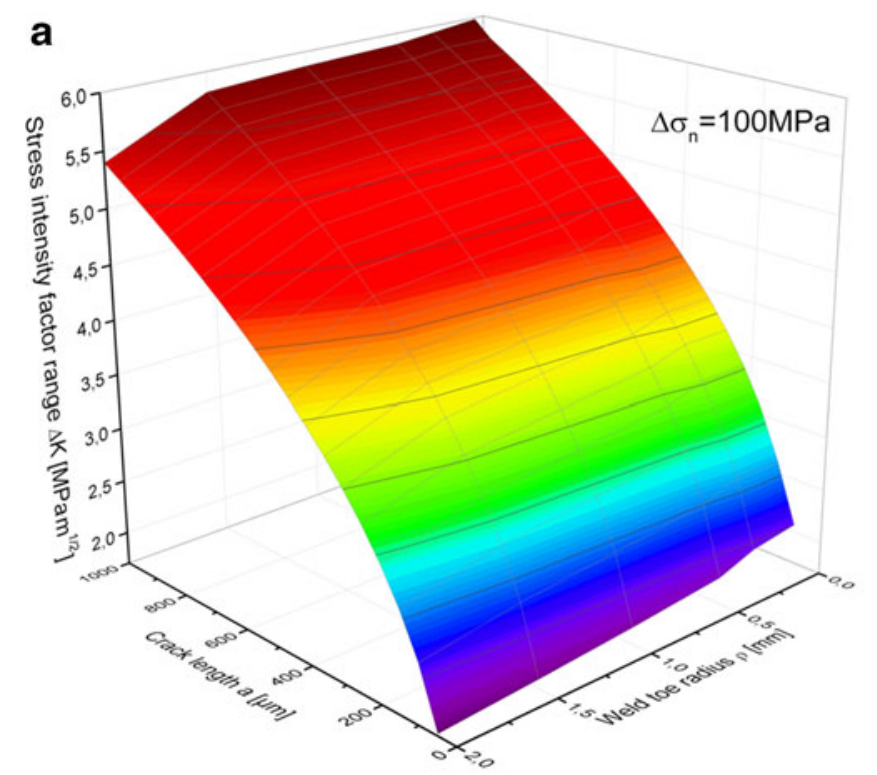

Summarized, an initial crack length of $a_{i}=0.1 \mathrm{~mm}$ and a weld toe radius of $\rho=0.05 \mathrm{~mm}$ lead to good accordance of the fatigue test results and are also in agreement with the recommended values [20]. These values are further on applied for the successive crack propagation analysis.

Figure 10a shows the effective stress ratio $R_{\text {eff }}$ at the crack tip in dependence of the stress ratio $R$ due to the loading and the actual crack length $a$. As the influence of the residual stress condition is considered by $R_{\mathrm{eff}}$, the effect of the residual stress distribution is clearly observable for the lowest stress ratio of $R=-1$. In this case, the compressive residual stress state at the surface layer of the weld toe region affects the local stress ratio $R_{\text {eff }}$ in a major way. If the crack propagates, the effect is

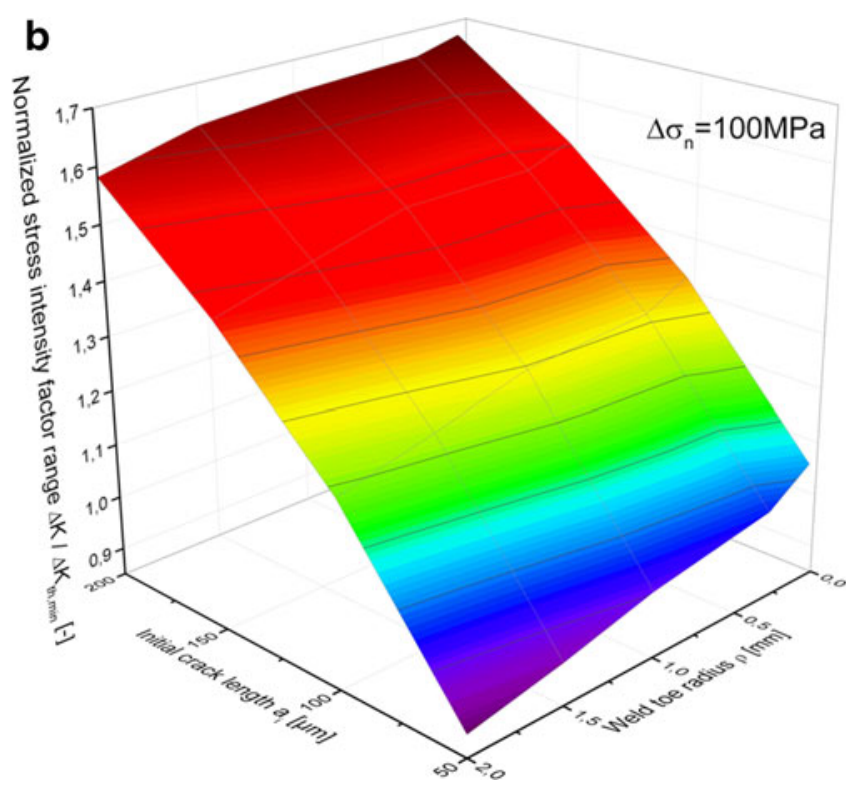

Fig. 9 a Stress intensity factor range $\Delta K$ during crack propagation and $\mathbf{b}$ ratio of $\Delta K / \Delta K_{\text {th,min }}$ in dependency of initial crack length $a_{i}$ 

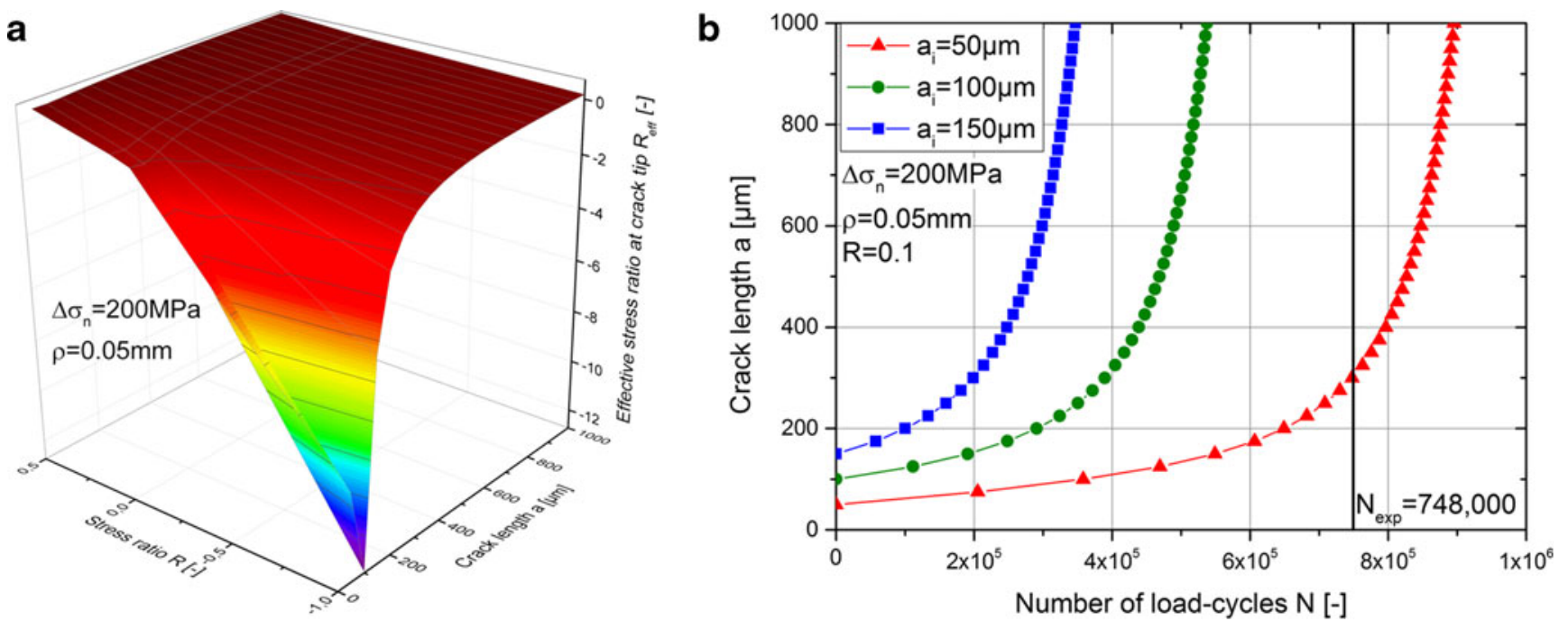

Fig. 10 a. Effective stress ratio $R_{\text {eff }}$ at crack tip and $\mathbf{b}$ calculated a/N curves for different initial crack lengths $a_{i}$

reduced and at a crack depth of about $a=0.5 \mathrm{~mm}$, no more influence is observable.

Otherwise, for high applied tumescent load-ratios of $R=0.5$, there is almost no effect of the residual stress condition on the local stress ratio at the crack tip. This is in accordance with the IIW-recommendation, whereas a fatigue testing at high mean stresses, preferably at $R=0.5$, should be conducted in order to minimize the influence of the weld process-induced residual stress condition.

In Fig. 10b, the resulting a/ $\mathrm{N}$ curves for a nominal stress range of $\Delta \sigma_{n}=200 \mathrm{MPa}$ and the applied stress ratio of $R=0.1$ are depicted for different initial crack lengths $a_{i}$. As already stated, an assumption of $a_{i}=0.05 \mathrm{~mm}$ may lead to an overestimation of the lifetime, which is also evaluated in this case. The recommended value of $a_{i}=0.1 \mathrm{~mm}$ calculates the lifetime in a safe but appropriate way, and further on, a definition of $a_{i}=0.15$ $\mathrm{mm}$ leads, as expected, to a conservative fatigue-life assessment.

\subsection{Parametric equation approach}

In case of simple weld geometries, such as butt welds, T-joints or longitudinal stiffeners, parametric formulae are provided [20, 29]. Thereby, the stress intensity factor $K$ is calculated according to Eq. 9 considering the crack-geometry-dependent correction function $Y(a)$ and an additional adjustment for the local notch of the weld toe $M_{k}(a)$. In [30], the magnification factor $M_{k}$ is presented, leading to a comparable stress condition for a crack of the same geometry, but without the presence of the weld notch. Furthermore, a separation of membrane $\sigma_{m}$ and shell bending stress $\sigma_{b}$ is necessary for most of the parametric formulae, whereas the resulting stress intensity factor $K$ is computed as a superposition of both stress components.

$K=\sqrt{\pi \cdot a} \cdot\left[\sigma_{m} \cdot Y_{m}(a) \cdot M_{k, m}(a)+\sigma_{b} \cdot Y_{b}(a) \cdot M_{k, b}(a)\right]$

In [20], the stress magnification factor $M_{k}$ for the end-ofseam region of a longitudinal stiffener is defined based on Eq. 10. Herein, the factor $C$ and exponent $k$ depend on the dimensions of the corresponding weld detail.

$M_{k}=C \cdot\left(\frac{a}{t}\right)^{k}$

Recent studies [31] involving a comparison of analytical, numerical and experimental results show good accordance and the basic applicability of the presented parametric formulae.

\subsection{Comparison of results}

To validate the fracture mechanical calculation, the calculated lifetime values are compared to the experimental fatigue test results, see Table 2 . Thereby, the initial crack length is set to
Table 2 Evaluated lifetime $N$ for different load-levels

\begin{tabular}{lllll}
\hline Initial crack length $a_{i}=0.1 \mathrm{~mm}$ & & & \\
\hline Nominal stress range $\Delta \sigma_{\mathrm{n}}[\mathrm{MPa}]$ & $N_{\exp }[-]$ & $\begin{array}{l}N_{W F}[-] \\
(\text { incl. RS) }\end{array}$ & $\begin{array}{l}N_{W F}[-] \\
(\text { excl. RS) }\end{array}$ & $\begin{array}{l}N_{P E}[-] \\
\text { (excl. RS) }\end{array}$ \\
\hline 100 & $2.1 \mathrm{e} 7$ & $1.5 \mathrm{e} 7$ & $1.3 \mathrm{e} 6$ & 194,000 \\
200 & 748,000 & 537,000 & 164,000 & 24,000 \\
300 & 95,000 & 100,000 & 48,000 & 7000 \\
\hline
\end{tabular}


the recommended value of $a_{i}=0.1 \mathrm{~mm}$ and the calculations are performed up to a final crack length of $a_{f}=1 \mathrm{~mm}$ because as shown in Fig. 4, within this crack propagation region, most of the lifetime is spent and just a minor remaining lifetime up to burst fracture is evaluated.

Firstly, a comparison of the experimental lifetime $N_{\text {exp }}$ and the calculated load-cycles by the weight-function approach $N_{W F}$ shows that by including the residual stress (RS) condition a well-matching fatigue assessment is deduced. On the contrary, the exclusion of the residual stress state leads to a significant reduction of the calculated lifetime, which can be explained by the mainly compressive residual stresses at the surface region maintaining a reduced crack propagation rate and, therefore, an increased lifetime. The effect of a superpositioned residual stress field on the crack propagation of surface cracks is principally analysed in [32].

Secondly, the application of the parametric equation approach to assess the fatigue-life exhibits the lowest and most conservative lifetime values $N_{P E}$. Also in this case, the beneficial compressive residual stresses are not considered, conducting to a major decrease for the lifetime estimation. However, one advantage of this method is the simple applicability with less effort and expenditure of time.

\section{Rehabilitation of pre-fatigued structures}

\subsection{Methods}

If a crack is detected or the service time of the structure or component is depleted, several rehabilitation methods are applicable. Simple retrofitting techniques are to attach bolted splices to the cracked parts or to achieve crack-growth arrest by drilling sop holes at the end of cracks [33]. A more elaborated way to restore cracked areas is fulfilled by re-welding. Especially wear-damaged sections of supporting structures are often weld-repaired, whereas an influence by the tri- or bimetal system on the fatigue crack growth should be analysed [34]. Another weld-repair technique which also improves the fatigue performance is based on the application of lowtemperature transformation welding wires. Thereby, compressive residual stress fields in the vicinity of the weld bead are introduced which has a beneficial effect on crack initiation and propagation and therefore enhances the fatigue strength [35].

Further technologies to rehabilitate pre-fatigued structures include the application of post-treatment methods. In [36], tungsten inert gas (TIG) and plasma dressing are applied to repair crack-containing $\mathrm{T}$-welded joints. It is shown that by TIG-dressing, only crack depths up to $2.5 \mathrm{~mm}$ can be successfully repaired. By the aid of plasma dressing, the results show an effect also for greater crack depths up to $4 \mathrm{~mm}$, whereas the fatigue-lives were found to be similar as for the as-welded condition. An overview of further available repair methods like the local explosive treatment, local heat treatment and high-frequency mechanical impact (HFMI) treatment with traditional techniques is given in [37]. However, based on the applicability and industrial feasibility, the HFMI treatment is one utmost efficient method, which is further on exemplified.

\subsection{HFMI treatment}

Not only for un-cracked but also for pre-fatigued joints, recent investigations [10, 38, 39] show a great beneficial effect for rehabilitation by the HFMI treatment, whereas the fatigue strength of the repaired specimens is equal or even exceeds the HFMI-treated condition without pre-cycling. As described, prefatigued specimens with a crack depth of $a \sim 1 \mathrm{~mm}$ are analysed to assess the potential of HFMI as a rehabilitation method without additional re-welding. A comparison of the evaluated fatigue test results is shown in Fig. 11. Thereby, the fatigue enhancement by the rehabilitation in the finite lifetime regime basically increases with a decreasing nominal load stress range. In the high-cycle fatigue region, the fatigue strength at the run-out level is almost identical to the HFMI-treated curve without pre-fatigue. Detailed information about the HFMI-treatment and fatigue tests is provided in [9].

The results are in good agreement with the previous investigations in literature, which generally confirm the applicability of the HFMI treatment as rehabilitation technique. One main influencing factor on the effectiveness is the crack length before performing the HFMI rehabilitation. In [10], it is shown, that for crack lengths above 1 to $2 \mathrm{~mm}$, a significant reduction of the benefit occurs, which is reasoned by the limited penetration depth by the HFMI treatment, depending on the local notch geometry, material properties and post-treatment parameters.

For the quantification of the rehabilitation benefit by the mechanical post-treatment, an advantageous gain factor $g_{N}$ is introduced in [10]. The value is defined as the ratio of the fatigue-life of the repaired joint $N_{r}$ to the expected fatiguelife of the joint if it was not repaired $N_{e}$, see Eqs. 11 and 12 .

$N_{r}=N_{i}+N_{b h}+N_{a H}$

$N_{e}=N_{i}+N_{b h}+N_{a H}^{\prime}$

Thereby, $N_{i}$ is the crack initiation life before the first crack for repair starts, $N_{b h}$ is the fatigue-life in crack propagation, $N_{a H}$ is the fatigue-life after repair including crack initiation and propagation stage, and $N_{a H}^{\prime}$ is the fatigue-life that would be obtained if the HFMI-repairtreatment would not be performed. An evaluation of the gain factor $g_{N}$ based on the experiments and accompanying crack initiation and propagation measurements indicates that the beneficial effect for a constant pre-crack length is depending on the applied load level, whereas for a higher nominal stress at $\Delta \sigma_{n}=300 \mathrm{MPa}$, the assessment 
Fig. 11 a/N curve of HFMIrehabilitated specimens

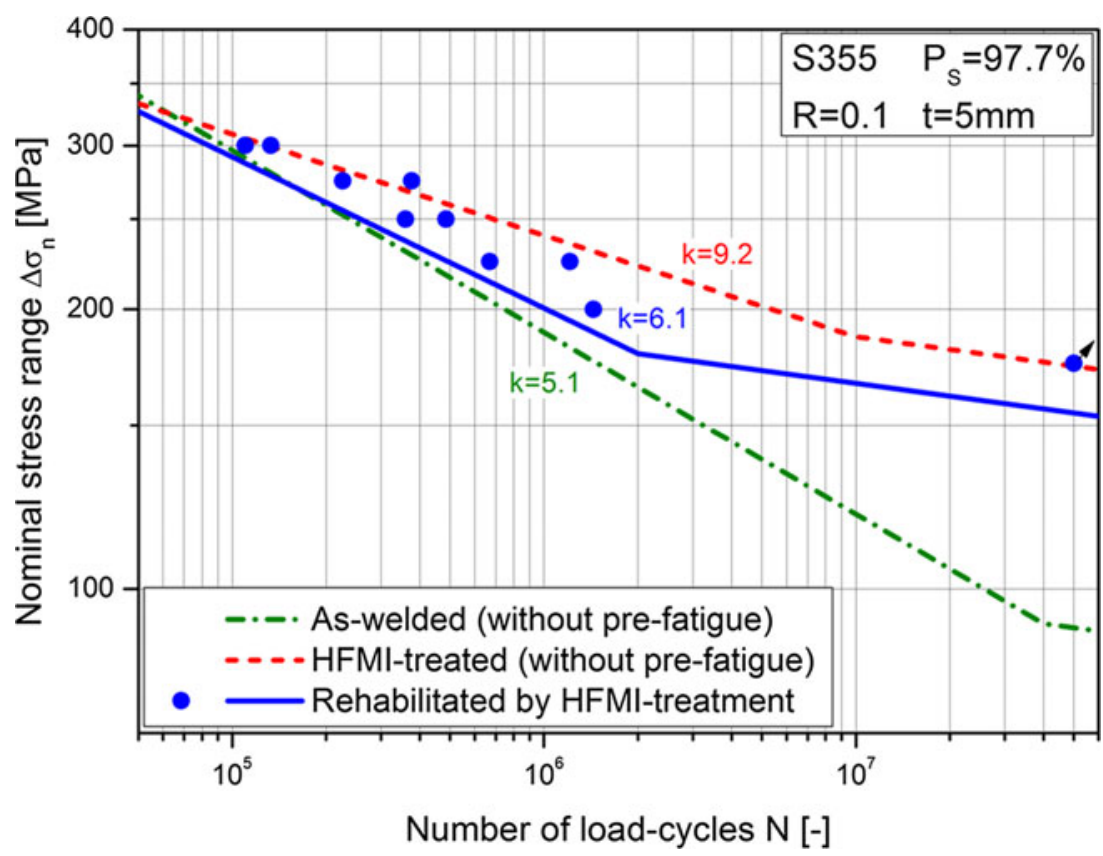

leads to $g_{N}=1.01$ and for a lower nominal stress at $\Delta \sigma_{n}=200 \mathrm{MPa}$ to $g_{N}=1.60$. The evaluated values are basically in good accordance to the results presented in [10]. Figure 12 presents an overview of the fatigue test data for HFMI-rehabilitated joints made of mild steel S355 considering the results of $[10,39]$ and this work (see Appendix). The comparison clearly shows that the beneficial effect of the HFMI treatment for pre-fatigued joints increases with a decrease of the nominal load.

Based on the investigated butt joint series in [39], it is concluded that a lifetime extension by the HFMI treatment is expected up to a maximum crack depth of $a=0.5 \mathrm{~mm}$. In [10], different initial crack depths and

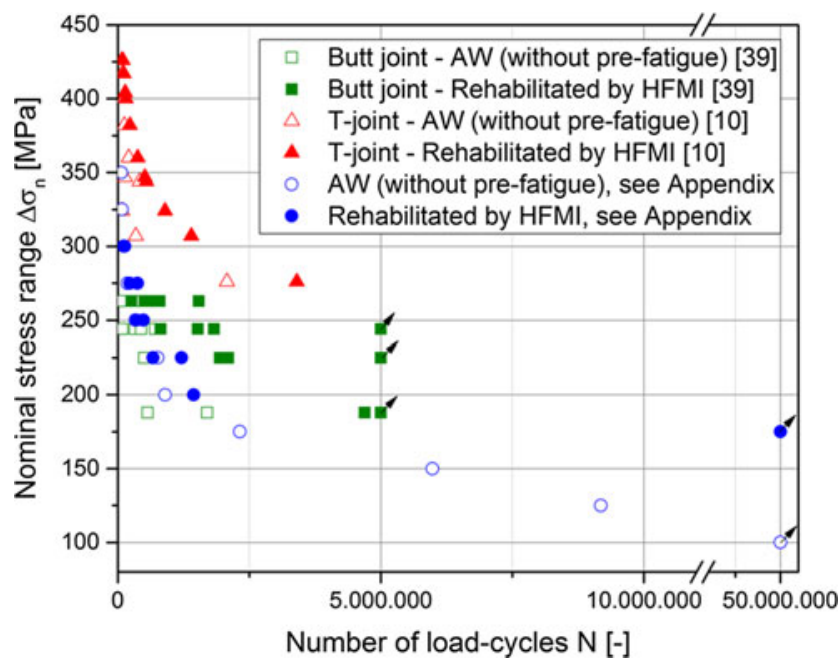

Fig. 12 Overview of fatigue test data for HFMI-rehabilitated mild steel joints $(R=0.1)$ their effect on the total lifetime after rehabilitation by HFMI is investigated, showing that the benefit is greater in repaired fatigue cracks with depths of $a \leq 2.5 \mathrm{~mm}$. However, several influences, such as structural weld detail, loading type, mean stress state, HFMI treatment parameters and compressive residual stress condition in depth, influence the effectiveness of the lifetime extension by HFMI. Considering the results in [10, 39] and by this work, a conservative maximum crack depth of $a_{\max }=0.5 \mathrm{~mm}$ can be recommended leading to an extension by the HFMI rehabilitation. In case of complex welded structures, an exact measurement of the crack length is not always possible maintaining an uncertainty of the applicability of the HFMI treatment. As exploited in [40], a re-welding and additional HFMI posttreatment as fatigue strengthening rehabilitation procedure is recommendable.

\section{Conclusion}

Crack propagation analysis of the investigated longitudinal stiffener based on the application of the weightfunction approach and considering the local residual stress condition leads to a good agreement with the evaluated fatigue test results. On the contrary, by neglecting the residual stress state, the deviation significantly increases towards a conservative design. The effect of weld process-induced residual stresses is essential for the analysed load-applied stress ratio of $R=0.1$ but diminishes at higher mean stress conditions. Additional numerical studies show a great influence of 
the weld toe radius, whereas an applied value of $\rho=0.05 \mathrm{~mm}$ exhibits appropriate results of the fracture mechanical calculations and matches well with the recommendation. A final comparison to the fracture mechanical standard approach based on parametric formulae shows that this method leads to a conservative assessment of the fatigue-life.

Fatigue test results in as-welded, HFMI-treated and precycled condition up to a crack depth of $a \sim 1 \mathrm{~mm}$ and subsequent HFMI repair indicate a beneficial effect of the mechanical post-treatment for pre-cracked joints. The fatigue strength after rehabilitation generally depends on the crack length before repair and furthermore on the applied load level, whereas the benefit increases with a decrease of the nominal stress range. Based on the own investigations and results within this work and by consideration of previously analyses regarding fatigue crack growth assessment and rehabilitation of prefatigued structures, the following conclusions can be drawn:

- The weight-function approach including an incorporation of the local residual stress condition is well applicable to assess the crack growth of welded joints based on the recommended values with a weld toe radius of $\rho=0.05 \mathrm{~mm}$ and an initial crack size of $a_{i}=0.1 \mathrm{~mm}$.

- An estimation of the crack size based on parametric formulae to calculate the stress intensity factor range $\Delta K$ shows a conservative fatigue assessment leading to an underrating of the lifetime up to a certain final crack length.

- On the basis of the results within this work, it can be concluded that if a structure is pre-fatigued and a certain crack size is detected, the remaining service life can be accurately and efficiently assessed based on the fracture mechanical weight-function approach.

- For a rehabilitation of pre-fatigued structures, the applied method significantly depends on the actual crack size. In general, a rehabilitation procedure involving grinding, rewelding and a final additional post-weld treatment is recommended. However, due to a limited time and cost frame, this elaborated routine cannot be completely fulfilled in industrial applications in some cases.

- If the HFMI treatment is applied without previous grinding and re-welding of the weld toe, a maximum crack size in depth of $a_{\max }=0.5 \mathrm{~mm}$ acts as conservative proposal for mild steel joints. For an application up to this crack depth value, the rehabilitation by HFMI leads to an improvement in fatigue strength both in the finite-life and high-cycle fatigue region. Due to slightly varying user-dependent treatment parameters and difference in obtained work-hardening, the applicability of this suggested maximum crack size cannot be thoroughly given for high-strength steels without any further specific investigations by the customer.

Acknowledgments Financial support by the Austrian Federal Government (in particular from Bundesministerium für Verkehr, Innovation und Technologie and Bundesministerium für Wirtschaft, Familie und Jugend) represented by Österreichische Forschungsförderungsgesellschaft $\mathrm{mbH}$ and the Styrian and the Tyrolean Provincial Government, represented by SteirischeWirtschaftsförderungsgesellschaft $\mathrm{mbH}$ and Standortagentur Tirol, within the framework of the COMET Funding Programme is gratefully acknowledged. Open access funding provided by Montanuniversität Leoben.

\section{Appendix}

Table 3 Fatigue test data points of investigated $\mathrm{a} / \mathrm{N}$-curves

\begin{tabular}{|c|c|c|c|c|c|}
\hline \multicolumn{2}{|c|}{ As-welded (without pre-fatigue) } & \multicolumn{2}{|c|}{ HFMI-treated (without pre-fatigue) } & \multicolumn{2}{|c|}{ Rehabilitated by HFMI treatmen } \\
\hline$\Delta \sigma_{\mathrm{n}}[\mathrm{MPa}]$ & $N[-]$ & $\Delta \sigma_{\mathrm{n}}[\mathrm{MPa}]$ & $N[-]$ & $\Delta \sigma_{\mathrm{n}}[\mathrm{MPa}]$ & $N[-]$ \\
\hline 350 & 67,808 & 350 & 101,314 & 300 & 133,000 \\
\hline 325 & 79,194 & 350 & 93,785 & 300 & 110,000 \\
\hline 300 & 131,791 & 325 & 169,677 & 275 & 377,000 \\
\hline 275 & 178,647 & 300 & 172,723 & 275 & 226,000 \\
\hline 250 & 335,957 & 275 & 565,548 & 250 & 485,000 \\
\hline 225 & 755,950 & 275 & 434,341 & 250 & 360,000 \\
\hline 200 & 900,086 & 250 & $2.81 \mathrm{E} 6$ & 225 & $1.21 \mathrm{E} 6$ \\
\hline 175 & $2.32 \mathrm{E} 6$ & 250 & $1.02 \mathrm{E} 6$ & 225 & 670,000 \\
\hline 150 & $5.98 \mathrm{E} 6$ & 225 & $6.90 \mathrm{E} 6$ & 200 & 1.44E6 \\
\hline 125 & $9.18 \mathrm{E} 6$ & 200 & $5 \mathrm{E} 7$ & 175 & $5 \mathrm{E} 7$ \\
\hline 100 & $5 \mathrm{E} 7$ & & & & \\
\hline
\end{tabular}


Open Access This article is distributed under the terms of the Creative Commons Attribution 4.0 International License (http:// creativecommons.org/licenses/by/4.0/), which permits unrestricted use, distribution, and reproduction in any medium, provided you give appropriate credit to the original author(s) and the source, provide a link to the Creative Commons license, and indicate if changes were made.

\section{References}

1. Hobbacher A (2009) IIW recommendations for fatigue design of welded joints and components, WRC bulletin 520. The Welding Research Council, New York

2. Hobbacher A (2011) Fracture and fatigue of welded joints and structures. Woodhead Publishing, Cambridge, pp. 91-112. http:// www.sciencedirect.com/science/book/9781845695132

3. Zerbst U, Ainsworth RA, Beier HT, Pisarski H, Zhang ZL, Nikbin K, Nitschke-Pagel T, Münstermann S, Kucharczyk P, Klingbeil D (2014) Review on fracture and crack propagation in weldments - a fracture mechanics perspective. Eng Fract Mech 132:200-276

4. Ditchburn RJ, Burke SK, Scala CM (1996) NDT of welds: state of the art. NDT\&E Int 29:111-117

5. Yildirim HC, Marquis GB (2012) Overview of fatigue data for high frequency mechanical impact treated welded joints. Weld World 56(7/8):82-96

6. Leitner M, Stoschka M, Eichlseder W (2014) Fatigue enhancement of thin-walled, high-strength steel joints by high-frequency mechanical impact treatment. Weld World 58:29-39

7. Marquis GB, Mikkola E, Yildirim HC, Barsoum Z (2013) Fatigue strength improvement of steel structures by high-frequency mechanical impact: proposed fatigue assessment guidelines. Weld World 57:803-822

8. Marquis GB, Barsoum Z (2014) Fatigue strength improvement of steel structures by high-frequency mechanical impact: proposed procedures and quality assurance guidelines. Weld World 58:19-28

9. Leitner M (2013) Local fatigue assessment of welded and high frequency mechanical impact treated joints, $\mathrm{PhD}$-thesis, Montanuniversität Leoben

10. Branco CM, Infante V, Baptista R (2004) Fatigue behaviour of welded joints with cracks, repaired by hammer peening. Fatigue Fract Eng Mater Struct 27:785-798

11. Shah F (2015) Crack propagation analysis of welded joints by numerical and experimental investigations, Master thesis, Montanuniversität Leoben

12. Fricke W (2008) Guideline for the fatigue assessment by notch stress analysis for welded structures, IIW-Document XIII-2240r108/XV-1289r1-08

13. Stoschka M, Ottersböck MJ, Leitner M (2014) Integration of phasedependent work-hardening into transient weld simulation, Proceedings of the Ninth International Conference on Engineering Computational Technology, Paper 39

14. ESI Group. (2014) SYSWELD Toolbox, release 16

15. Prager W (1949) Recent development in the mathematical theory of plasticity J Appl Phys vol. 235, issue 7

16. Armstrong P, Frederick C (1966) A mathematical representation of the multiaxial Bauschinger effect, CEGB Report RD/B/N731

17. Chaboche J-L (2008) A review of some plasticity and viscoplasticity constitutive theories. Int J Plast 24:1642-1693

18. Ottersböck M, Stoschka M, Thaler M (2013) Study of kinematic strain hardening law in transient welding simulation. Math Model Weld Phenom 10:255-266
19. Fricke W (2003) Fatigue analysis of welded joints: state of development. Mar Struct 16:185-200

20. Hobbacher A (2012) Update of the fracture mechanics chapters of the IIW fatigue design recommendations, IIW-document XIII2370r1-11 / XV-1376r1-11

21. Paris P, Erdogan F (1963) A critical analysis of crack propagation laws, Journal of Basic Engineering. Trans Am Soc Mech Eng 85(4): 528-534

22. Murakami Y.: Stress intensity factors handbook, Pergamon, Oxford, vol. 2, 1987

23. Bueckner HF (1970) A novel principle for the computation of stress intensity factors. Z Angew Math Mech 50:529-546

24. Glinka G, Shen G (1991) Universal features of weight functions for cracks in mode I. Eng Fract Mech 40(6):1135-1146

25. Chattopadhyay A, Glinka G, El-Zein M, Qian J, Formas R (2011) Stress analysis and fatigue of welded structures. Weld World 55(7/8):2-21

26. Goyal R, Glinka G (2013) Fracture mechanics-based estimation of fatigue lives of welded joints. Weld World 57:625-634

27. Glinka G.: Effect of residual stresses on fatigue crack growth in steel weldments under constant and variable amplitude loading, Fracture Mechanics, ASTM STP 677, American Society for Testing and Materials, pp. 198-214, 1979

28. Kurihara M, Katoh A, Kwaahara M (1986) Analysis on fatigue crack growth rates under a wide range of stress ratio. J Press Vessel Technol, Trans ASME 108(2):209-213

29. Hobbacher A (1993) Stress intensity factors of welded joints. Engineering Fracture Mechanics 46(2):173-182, and vol. 49, no. 2, p. 323, 1994

30. Maddox SJ (1975) An analysis of fatigue cracks in fillet welded joints. Int J Fract 11(2):221-243

31. Al-Mukhtar AM, Henkel S, Biermann H, Hübner P (2009) A finite element calculation of stress intensity factors of cruciform and butt welded joints for some geometrical parameters. Jordan J Mech Ind Eng 3(4):236-245

32. Mochizuki M, Miyazaki K (2006) Surface crack propagation analysis under residual stress field. Weld World 50(5/6):38-45

33. Miki C (2008) Retrofitting engineering for fatigue damaged steel structures, IIW-document XIII-2284-09 / WG5-74-07, revised 2008

34. Zhang C, van der Vyver S, Hua X, Lu P (2011) Fatigue crack growth behavior in weld-repaired high-strength low-alloy steel. Eng Fract Mech 78:1862-1875

35. Miki C, Hanji T, Tokunaga K (2012) Weld repair for fatiguecracked joints in steel bridges by applying low temperature transformation welding wire. Weld World 56(3/4):40-50

36. Ramalho A, Ferreira J, Branco C (2011) Fatigue behaviour of T welded joints rehabilitated by tungsten inert gas and plasma dressing. Mater Des 32:4705-4713

37. Kudryavtsev Y., Kleiman J., Knysh V., Mikheev P.: Fatigue life improvement of structural elements with fatigue cracks, Proceedings of the SEM Annual Conference \& Exposition on Experimental and Applied Mechanics, 2002

38. Kudryavtsev Y, Kleiman J, Lobanov L, Knysh V, Voitenko O, Prokopenko G, Lugovskoy A (2007) Rehabilitation and repair of welded elements and structures by ultrasonic peening. Weld World 51(7/8):47-43

39. Weich I (2009) Ermüdungsverhalten mechanisch nachbehandelter Schweißverbindungen in Abhängigkeit des Randschichtzustands (Fatigue behaviour of mechanical post weld treated welds depending on the edge layer condition), $\mathrm{PhD}$-thesis, Technische Universität Braunschweig, in German

40. Tominaga T, Matsuoka K, Sato Y, Suzuki T (2008) Fatigue improvement of weld repaired crane runway girder by ultrasonic impact treatment. Weld World 52(11/12):50-62 